\title{
Explaining the link between access-to-care factors and health care resource utilization among individuals with COPD
}

This article was published in the following Dove Press journal:

International Journal of COPD

22 February 2016

Number of times this article has been viewed

\author{
Minchul Kim' \\ Jinma Ren' \\ William Tillis ${ }^{2,3}$ \\ Carl V Asche ${ }^{1,4}$ \\ Inkyu K Kim ${ }^{5}$ \\ Carmen S Kirkness' \\ 'Department of Internal Medicine, \\ Center for Outcomes Research, \\ University of Illinois College of \\ Medicine at Peoria, ${ }^{2}$ OSF St Francis \\ Medical Center, ${ }^{3}$ Department of \\ Internal Medicine, University of \\ Illinois College of Medicine at Peoria, \\ Peoria, ${ }^{4}$ Department of Pharmacy \\ Systems, Outcomes and Policy, \\ University of Illinois at Chicago \\ College of Pharmacy, Chicago, IL, \\ ${ }^{5}$ Battelle Memorial Institute, Atlanta, \\ GA, USA
}

Background: Limited accessibility to health care may be a barrier to obtaining good care. Few studies have investigated the association between access-to-care factors and COPD hospitalizations. The objective of this study is to estimate the association between access-tocare factors and health care utilization including hospital/emergency department (ED) visits and primary care physician (PCP) office visits among adults with COPD utilizing a nationally representative survey data.

Methods: We conducted a pooled cross-sectional analysis based upon a bivariate probit model, utilizing datasets from the 2011-2012 Behavioral Risk Factor Surveillance System linked with the 2014 Area Health Resource Files among adults with COPD. Dichotomous outcomes were hospital/ED visits and PCP office visits. Key covariates were county-level access-to-care factors, including the population-weighted numbers of pulmonary care specialists, PCPs, hospitals, rural health centers, and federally qualified health centers.

Results: Among a total of 9,332 observations, proportions of hospital/ED visits and PCP office visits were $16.2 \%$ and $44.2 \%$, respectively. Results demonstrated that access-to-care factors were closely associated with hospital/ED visits. An additional pulmonary care specialist per 100,000 persons serves to reduce the likelihood of a hospital/ED visit by 0.4 percentage points (pp) $(P=0.028)$. In contrast, an additional hospital per 100,000 persons increases the likelihood of hospital/ED visit by $0.8 \mathrm{pp}(P=0.008)$. However, safety net facilities were not related to hospital utilizations. PCP office visits were not related to access-to-care factors.

Conclusion: Pulmonary care specialist availability was a key factor in reducing hospital utilization among adults with COPD. The findings of our study implied that an increase in the availability of pulmonary care specialists may reduce hospital utilizations in counties with little or no access to pulmonary care specialists and that since availability of hospitals increases hospital utilization, directing patients with COPD to pulmonary care specialists may decrease hospital utilizations.

Keywords: pulmonary specialist, COPD, hospital utilization

\section{Introduction}

Chronic respiratory disease including COPD represents a considerable burden in the USA, ranking third out of all causes of death in $2010 .{ }^{1}$ The total economic burden of COPD was predicted to be $\$ 52.7$ billion (in 2015 US dollars), which included direct medical costs ( $\$ 32.4$ billion), indirect morbidity costs ( $\$ 10.4$ billion), and premature mortality costs ( $\$ 9.9$ billion). ${ }^{2}$ Hospitalization and emergency department (ED) costs represented $72.8 \%$ of direct medical costs. ${ }^{2}$ They consisted of relatively low frequencies and high per-visit cost as compared to that of primary care physician (PCP) office visits. ${ }^{3}$ COPD is also associated with an increase of cost burden over time. ${ }^{4}$
Correspondence: Minchul Kim

Department of Internal Medicine, Center for Outcomes Research, University of Illinois College of Medicine at Peoria, One Illini Drive, Peoria, IL 61656-1649, USA

$\mathrm{Tel}+|30967| 8429$

Fax +I $30967 \mid 8438$

Email mchkim@uic.edu
International Journal of COPD 2016: I 357-367

357

Dovepress

http://dx.doi.org/10.2147/COPD.S95717 (c) (1) (5) $2016 \mathrm{Kim}$ et al. This work is published and licensed by Dove Medical Press Limited. The full terms of this license are available at https://www.dovepress.com/terms.php
and incorporate the Creative Commons Attribution - Non Commercial (unported, v3.0) License (http://(creativecommons.org/licenses/by-nc/3.0/). By accessing the work you BY NC
hereby accept the Terms. Non-commercial uses of the work are permitted without any further permission from Dove Medical Press Limited, provided the work is properly attributed. For permission for commercial use of this work, please see paragraphs 4.2 and 5 of our Terms (https://www.dovepress.com/terms.php).
her 
A Healthy People 2020 target for COPD is to reduce hospitalizations and $\mathrm{ED}$ visits among patients with COPD. ${ }^{5}$ A great strategy to reduce utilization of hospitals and EDs is to prevent acute exacerbations among patients with COPD since the acute exacerbation is a major cause of COPD hospitalization and ED visits. ${ }^{6}$ Improving treatment and management of patients with COPD will prevent acute exacerbations, leading to reduction of hospitalizations or ED visits. ${ }^{7}$

As determinants of hospitalizations among patients with COPD, previous studies only focused on patient's characteristics, including demographic/socioeconomic factors, health status factors, and therapeutic history to determine populations vulnerable to hospitalization. ${ }^{8-13}$ Some studies examined the environmental factors such as air quality and smoking-free public policy. ${ }^{14-16}$ Yet, few studies have investigated the associations between health status/hospitalizations and availability/accessibility of access-to-care factors such as PCP, safety net facilities, pulmonary specialists, and hospitals..$^{17,18}$

Availability/accessibility of access-to-care factors is becoming increasingly more important among patients since limited accessibility is becoming a barrier to receive good care. ${ }^{19}$ Limited availability/accessibility of access-to-care factors may cause diverse effects on patients' health, leading to increased or decreased hospitalizations. ${ }^{20,21} \mathrm{~A}$ key accessto-care factor for patients with COPD might be pulmonary care specialists who can provide better treatment and management to patients with COPD than that of general physicians, leading to reductions in health care utilizations..$^{22,23}$ Safety net facilities, such as rural health centers (RHCs) and federally qualified health centers (FQHCs), provided primary care among financially disadvantaged populations, leading to reducing ambulatory care sensitive conditions and, consequently, those became an access-to-care factor. ${ }^{24,25}$ Hospitals and PCPs might be access-to-care factors as there are limited numbers of hospitals and PCPs, thus becoming a barrier to access due to the long distance required to travel to hospitals and PCPs. ${ }^{26}$

Jackson et $\mathrm{a}^{17}$ found an inverse relationship between the availability of pulmonary care specialist and hospitalizations, utilizing Texas Health Care Information Council inpatient data. However, to our knowledge, there were no studies on associations between access-to-care factors and hospitalizations among patients with COPD utilizing a nationwide sample. The objective of our study is to estimate the association between access-to-care factors and health care utilizations including hospital/ED visits and PCP office visits among adults with COPD utilizing a nationally representative survey data. Access-to-care factors will include pulmonary care specialists, safety net facilities, hospitals, and PCPs. Clarifying the relationship between health care utilization and the availability of resources will assist in developing new policies targeted to reduce hospitalization burden.

\section{Materials and methods Datasets}

We analyzed datasets of the 2011-2012 Behavioral Risk Factor Surveillance System (BRFSS) ${ }^{27}$ linked with the 2014 Area Health Resource Files (AHRF) ${ }^{28}$ BRFSS is a nationally representative survey dataset, which commenced in 1984 to collect behavioral health risk data via telephone interviews among adults (18+ years old), covering all 50 states, District of Columbia, and three US territories. In our study, we extracted the COPD-related information from BRFSS including ED/hospital visits and PCP office visits among adults with COPD. COPD was diagnosed based upon a survey question asking whether a subject has COPD, emphysema, or chronic bronchitis.

The 2014 AHRF dataset was linked with BRFSS dataset based upon state and county variables. AHRF provides $>6,000$ variables on health resources and socioeconomic characteristics, including health facilities and health professions at the county level. For the purpose of our study, the population-weighted numbers of pulmonary care specialists, PCPs, hospitals, RHCs, and FQHCs were obtained from AHRF.

\section{Study population}

The final sample from our data analysis included 9,332 observations as depicted in Figure 1. We extracted 77,267 observations of adults with COPD from the dataset. We then excluded those who had missing values in terms of outcome variables $(69,298$ observations), women with pregnancy (18 observations), and those with missing access-to-care factors (1,057 observations) and other covariates $(2,562$ observations).

\section{Statistical analysis}

We conducted a pooled cross-sectional analysis utilizing a bivariate probit model, ${ }^{29}$ where dichotomous outcomes were determined to be indicators on 1) COPD-related ED or hospital visits and 2) COPD-related PCP office visit. The bivariate probit model was employed since we found a positive correlation (coefficient of correlation $=0.40, P<0.001$ ) between the two outcome variables (Table S1). In addition the estimate of rho $(\rho)$ in the bivariate probit model depicted 


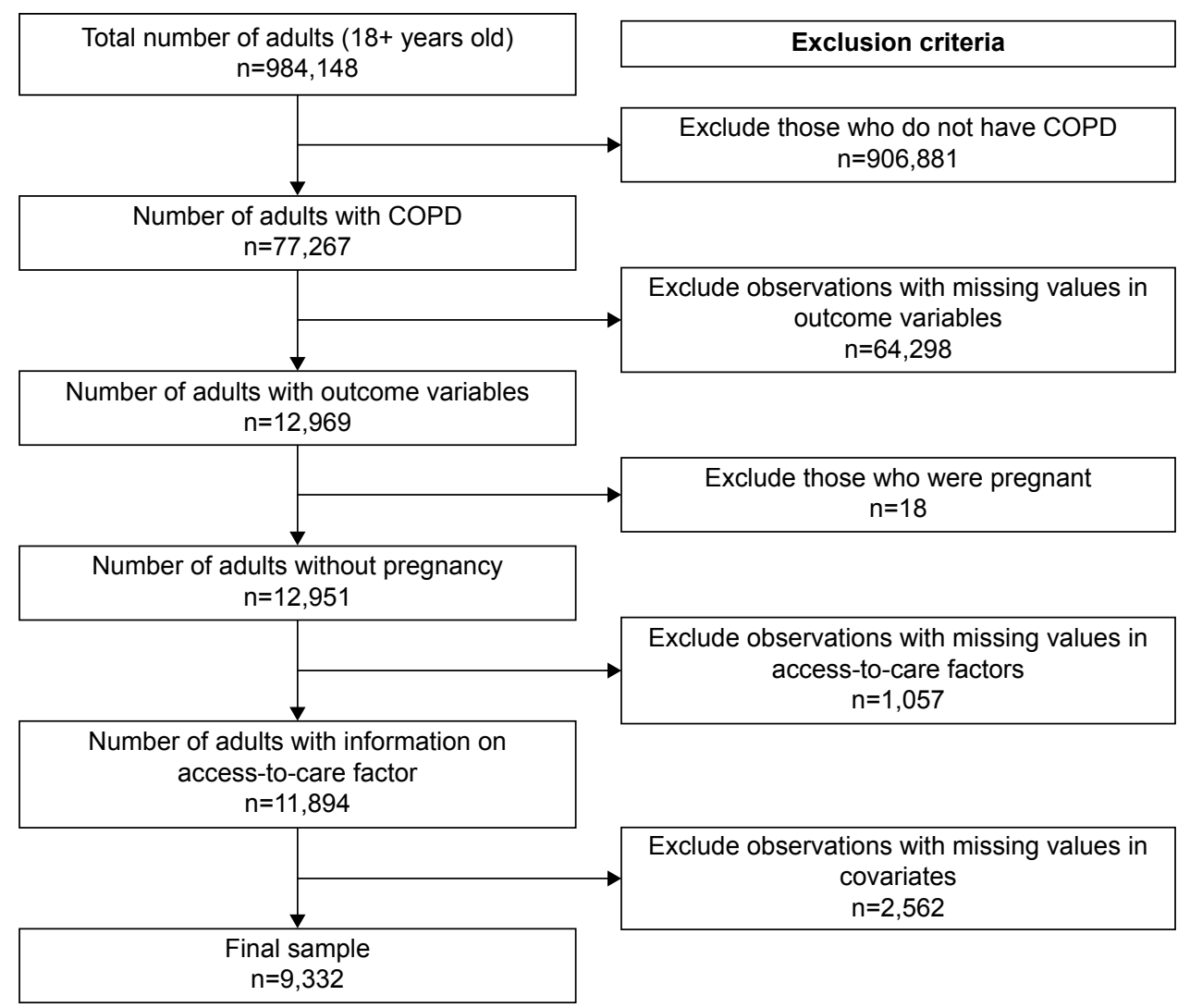

Figure I Sample extraction.

in Table 2 was $0.69(P<0.001)$, indicating that unobservable factors were positively associated with both outcome variables. We obtained the marginal effects of all variables at the mean values in order to determine the percentage point (pp) change of the likelihood of outcomes per unit change of covariate.

Key covariates were county-level access-to-care factors. For the first outcome (hospital/ED visit), the number of pulmonary care specialists per 100,000 persons, the number of safety net facilities per 100,000 persons, including FQHCs and RHCs, and the number of hospitals per 100,000 persons were included. For the second outcome (PCP office visit), the number of pulmonary care specialists per 100,000 persons, the number of safety net facilities per 100,000 persons, and the number of PCPs per 10,000 persons were included. RHCs and FQHCs were federally qualified health clinics certified to receive special Medicare and Medicaid reimbursement and introduced to provide vulnerable population with primary care ${ }^{17}$ RHCs should be located in nonurban area, while FQHCs have no restrictions. ${ }^{17}$

All county-level variables were available from 2011 to 2012 in AHRF except for the number of hospitals, which were available only in 2010 and 2011. As such, the 2012 year data for the number of hospitals were obtained via extrapolation methods based upon 2010 and 2011 data. All variables were divided by county-level population.

Other covariates for both outcomes included age-group, sex, race, marital status, education level, employment status, smoking status, income, health insurance coverage, COPDmedications, dichotomous indicators on comorbidities (myocardial infarction, angina or coronary heart disease, stroke, asthma, cancer, arthritis, depression, kidney disease, and diabetes), region, and year.

Since variables for access-to-care factors may be effective on outcomes after a year, we conducted statistical analyses based upon lagged values of these variables as a sensitivity analysis. We utilized previous year values for these variables in order to verify whether the result of primary analysis was sensitive to the lagged values. We also conducted a subpopulation analysis based upon the presence of pulmonary care specialists because the presence of pulmonary care specialists may impact the effects of other factors on health care utilizations.

All analyses in this study were conducted using STATA version 12 (StataCorp LP, College Station, TX, USA). The significance level was set to 0.05 . Due to complex sampling 
procedures, we used the survey command function to conduct weighted regression analyses.

\section{Results}

Among 9,332 adults with COPD, less than half (43\%) were male and older age-groups shared higher proportions than younger age-groups; from 11.2\% (aged 18-34 years) to $24.9 \%-32.3 \%$ (aged $55+$ years). The proportions of
hospital/ED visits and PCP office visits were 16.2\% and $44.2 \%$, respectively (Table 1 ). A positive relationship between two outcomes was found. For example, those with PCP office visits were 30.6 pp more likely to have hospital/ ED visits $(P<0.001)$. Access-to-care factors were not different between those with health care utilizations and those without, except for the number of hospitals. Those with hospital/ED visits had a few more hospitals within counties

Table I Descriptive statistics: unweighted/weighted N=9,332/2,09।,774

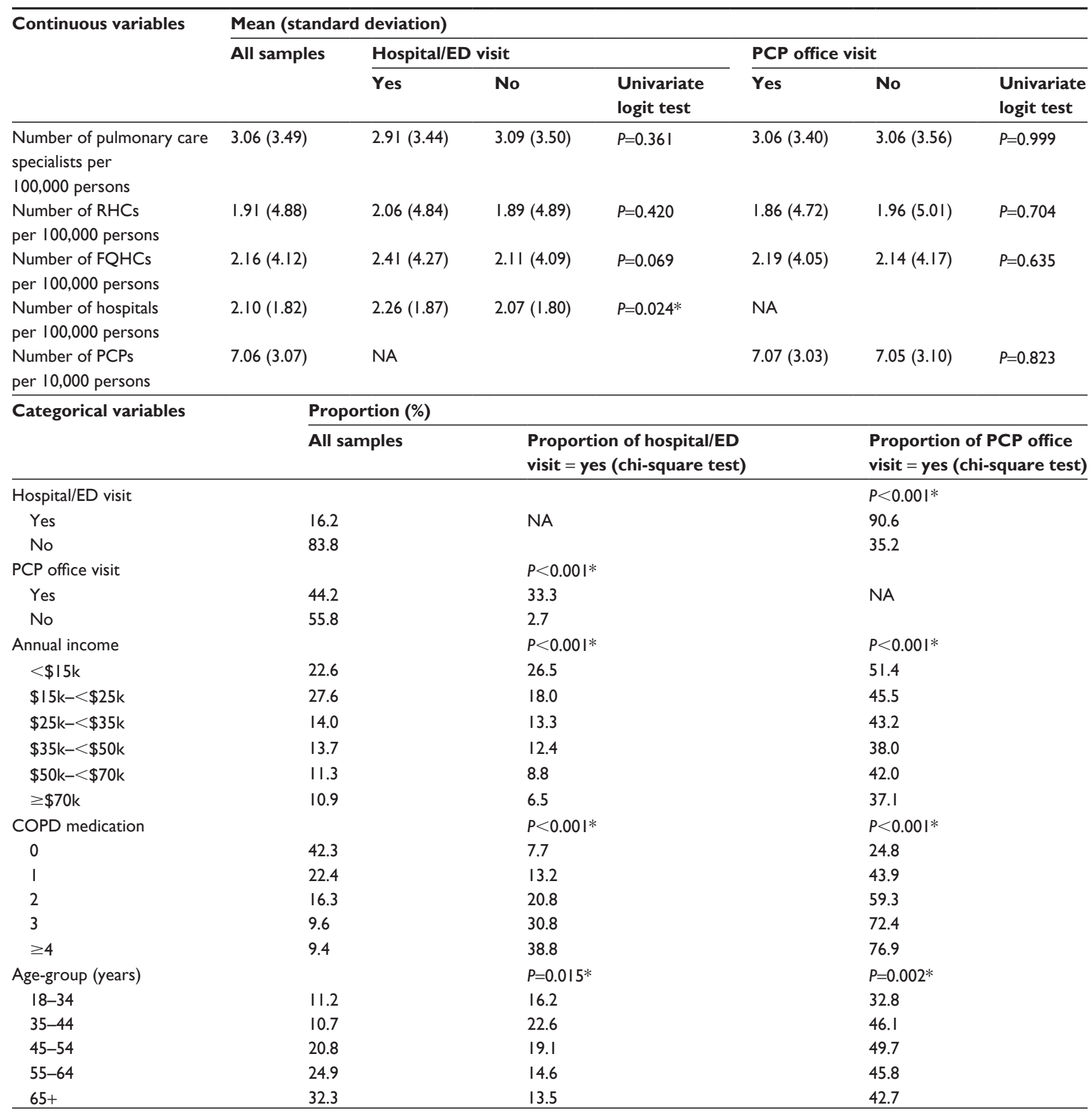


Table I (Continued)

\begin{tabular}{|c|c|c|c|}
\hline \multirow[t]{2}{*}{ Categorical variables } & \multicolumn{3}{|c|}{ Proportion (\%) } \\
\hline & All samples & $\begin{array}{l}\text { Proportion of hospital/ED } \\
\text { visit = yes (chi-square test) }\end{array}$ & $\begin{array}{l}\text { Proportion of PCP office } \\
\text { visit }=\text { yes (chi-square test) }\end{array}$ \\
\hline \multicolumn{2}{|l|}{ Sex } & $P=0.048^{*}$ & $P<0.00 I^{*}$ \\
\hline Male & 43.1 & 14.3 & 37.9 \\
\hline Female & 56.9 & 17.6 & 49.0 \\
\hline \multicolumn{2}{|l|}{ Race } & $P<0.00 I^{*}$ & $P=0.005^{*}$ \\
\hline Caucasian & 77.6 & 13.9 & 42.1 \\
\hline African-American & 11.7 & 28.2 & 54.2 \\
\hline Hispanic & 4.7 & 18.3 & 41.4 \\
\hline Other races & 5.9 & 21.7 & 53.7 \\
\hline \multicolumn{2}{|l|}{ Marital status } & $P=0.02 I^{*}$ & $P=0.115$ \\
\hline Married & 48.9 & 14.1 & 44.3 \\
\hline Single & 14.8 & 18.6 & 40.5 \\
\hline Divorced & 20.2 & 20.1 & 48.1 \\
\hline Widowed & 12.9 & 14.8 & 43.6 \\
\hline Separated & 3.1 & 18.4 & 37.2 \\
\hline \multicolumn{2}{|l|}{ Education } & $P<0.00 I^{*}$ & $P=0.202$ \\
\hline Some high school & 22.6 & 22.0 & 47.9 \\
\hline High-school graduation & 34.7 & 15.8 & 43.3 \\
\hline Some college & 30.7 & 14.3 & 42.9 \\
\hline College graduation & 12.0 & 11.4 & 43.1 \\
\hline \multicolumn{2}{|l|}{ Employment } & $P<0.00 I^{*}$ & $P=0.003^{*}$ \\
\hline Yes & 30.7 & II.I & 39.5 \\
\hline No & 69.3 & 18.5 & 46.3 \\
\hline \multicolumn{2}{|l|}{ Smoking status } & $P=0.577$ & $P=0.008^{*}$ \\
\hline Nonsmoker & 59.0 & 16.6 & 46.5 \\
\hline Current smoker & 41.0 & 15.7 & 40.9 \\
\hline \multicolumn{2}{|l|}{ Myocardial infarction } & $P<0.00 I^{*}$ & $P<0.00$ I* \\
\hline Yes & 16.6 & 26.7 & 56.4 \\
\hline No & 83.4 & 14.1 & 41.8 \\
\hline \multicolumn{2}{|c|}{ Angina or coronary heart disease } & $P<0.00 I^{*}$ & $P<0.00$ I* \\
\hline Yes & 16.8 & 24.6 & 56.3 \\
\hline No & 83.2 & 14.5 & 41.8 \\
\hline \multicolumn{2}{|l|}{ Stroke } & $P<0.00 I^{*}$ & $P=0.073$ \\
\hline Yes & 8.9 & 23.1 & 49.1 \\
\hline No & 91.1 & 15.6 & 43.7 \\
\hline \multicolumn{2}{|l|}{ Asthma } & $P<0.00 I^{*}$ & $P<0.00 I *$ \\
\hline Yes & 42.9 & 20.8 & 54.2 \\
\hline No & 57.1 & 12.8 & 36.7 \\
\hline \multicolumn{2}{|l|}{ Cancer } & $P=0.823$ & $P=0.007^{*}$ \\
\hline Yes & 21.9 & 16.5 & 48.4 \\
\hline No & 78.1 & 16.1 & 43.0 \\
\hline \multicolumn{2}{|l|}{ Arthritis } & $P=0.233$ & $P<0.00 I^{*}$ \\
\hline Yes & 57.1 & 17.0 & 48.4 \\
\hline No & 42.9 & 15.2 & 38.5 \\
\hline \multicolumn{2}{|l|}{ Depression } & $P<0.00 I^{*}$ & $P<0.00 I^{*}$ \\
\hline Yes & 39.2 & 19.8 & 50.1 \\
\hline No & 60.8 & 13.9 & 40.4 \\
\hline \multicolumn{2}{|l|}{ Kidney disease } & $P<0.00 I^{*}$ & $P=0.003^{*}$ \\
\hline Yes & 7.0 & 24.7 & 54.2 \\
\hline No & 93.0 & 15.6 & 43.4 \\
\hline \multicolumn{2}{|l|}{ Diabetes } & $P=0.004^{*}$ & $P<0.00 I^{*}$ \\
\hline Yes & 20.0 & 20.2 & 52.1 \\
\hline No & 80.0 & 15.2 & 42.2 \\
\hline
\end{tabular}

Notes: Region, health care coverage, and year variables were not included in Table I. $* P<0.05$.

Abbreviations: ED, emergency department; RHCs, rural health centers; FQHCs, federally qualified health centers; NA, not applicable; PCP, primary care physician. 
as compared to those without hospital/ED visits $(P=0.024)$. Usage of COPD medications was positively related to the proportions of hospital/ED visits and PCP office visits. Increasing income level was negatively related to the proportions of hospital/ED visits.
As listed in Table 2, our primary analysis determined that access-to-care factors were closely associated with hospital/ ED visits rather than PCP office visits. The availabilities of pulmonary care specialists and hospitals were negatively and positively associated with hospital utilizations,

Table 2 Results of bivariate probit model with two outcomes (hospital/ED visit and PCP office visit) (unweighted/weighted $\mathrm{N}=9,332 / 2,091,774)$

\begin{tabular}{|c|c|c|c|c|c|c|}
\hline \multirow{3}{*}{$\begin{array}{l}\text { Outcomes } \\
\text { Covariates (reference group) }\end{array}$} & \multicolumn{6}{|c|}{ Bivariate probit model (unweighted/weighted $\mathrm{N}=9,332 / 2,091,774$ ) } \\
\hline & \multicolumn{3}{|c|}{ Hospital/ED visit } & \multicolumn{3}{|c|}{ PCP office visit } \\
\hline & Coefficient & P-value & Marginal effect & Coefficient & $P$-value & Marginal effect \\
\hline Pulmonary care specialists per & -0.019 & $0.028 *$ & -0.004 & -0.007 & 0.461 & -0.002 \\
\hline \multicolumn{7}{|l|}{100,000 persons } \\
\hline RHCs per 100,000 persons & -0.003 & 0.704 & -0.001 & -0.002 & 0.637 & -0.001 \\
\hline FQHCs per 100,000 persons & 0.007 & 0.401 & 0.001 & 0.003 & $0.74 I$ & 0.001 \\
\hline Hospitals per 100,000 persons & 0.037 & $0.032^{*}$ & 0.008 & NA & & \\
\hline PCPs per 10,000 persons & NA & & & 0.002 & 0.872 & 0.001 \\
\hline \multicolumn{7}{|l|}{ Annual income $(\$ 35 k-<\$ 50 k)$} \\
\hline$<\$ 15 k$ & 0.266 & $0.02 I^{*}$ & 0.060 & 0.174 & 0.071 & 0.058 \\
\hline$\$ 15 k-<\$ 25 k$ & 0.115 & 0.282 & 0.024 & 0.140 & 0.091 & 0.046 \\
\hline$\$ 25 k-<\$ 35 k$ & -0.018 & 0.869 & -0.004 & 0.116 & 0.183 & 0.038 \\
\hline$\$ 50 \mathrm{k}-<\$ 70 \mathrm{k}$ & -0.150 & 0.188 & -0.028 & 0.109 & 0.233 & 0.036 \\
\hline$\geq \$ 70 \mathrm{k}$ & -0.390 & $0.003^{*}$ & -0.065 & -0.067 & 0.518 & -0.022 \\
\hline \multicolumn{7}{|l|}{ COPD medication (no medication) } \\
\hline 1 & 0.307 & $<0.00 I^{*}$ & 0.051 & 0.519 & $<0.00 I^{*}$ & 0.181 \\
\hline 2 & 0.639 & $<0.00 I^{*}$ & 0.128 & 0.892 & $<0.00 I^{*}$ & 0.323 \\
\hline 3 & 0.988 & $<0.00 I^{*}$ & 0.232 & 1.251 & $<0.00 I^{*}$ & 0.450 \\
\hline$\geq 4$ & 1.099 & $<0.00 I^{*}$ & 0.270 & 1.362 & $<0.001 *$ & 0.485 \\
\hline Health care coverage & -0.150 & 0.122 & -0.031 & 0.065 & 0.462 & 0.021 \\
\hline \multicolumn{7}{|l|}{ Age-group (18-34) } \\
\hline $35-44$ & -0.057 & 0.699 & -0.015 & 0.076 & 0.576 & 0.025 \\
\hline $45-54$ & -0.166 & 0.180 & -0.041 & 0.139 & 0.221 & 0.046 \\
\hline $55-64$ & -0.441 & $0.00 I^{*}$ & -0.099 & -0.077 & 0.490 & -0.026 \\
\hline $65+$ & -0.521 & $<0.00 I^{*}$ & -0.114 & -0.221 & 0.072 & -0.072 \\
\hline Male & -0.172 & $0.007^{*}$ & -0.036 & -0.313 & $<0.001 *$ & -0.104 \\
\hline \multicolumn{7}{|l|}{ Race (Caucasian) } \\
\hline African-American & 0.393 & $<0.00 I^{*}$ & 0.092 & 0.253 & $0.009 *$ & 0.085 \\
\hline Hispanic & 0.123 & 0.542 & 0.026 & 0.038 & 0.812 & 0.013 \\
\hline Other races & 0.126 & 0.323 & 0.027 & 0.160 & 0.111 & 0.054 \\
\hline \multicolumn{7}{|l|}{ Marital status (married) } \\
\hline Single & -0.123 & 0.298 & -0.025 & -0.127 & 0.146 & -0.042 \\
\hline Divorced & -0.060 & 0.456 & -0.013 & -0.125 & 0.064 & -0.041 \\
\hline Widowed & -0.173 & $0.029 *$ & -0.035 & -0.240 & $<0.001 *$ & -0.079 \\
\hline Separated & -0.200 & 0.173 & -0.040 & -0.422 & $0.003^{*}$ & -0.136 \\
\hline \multicolumn{7}{|l|}{ Education (some high school) } \\
\hline High-school graduation & -0.099 & 0.222 & -0.021 & -0.081 & 0.271 & -0.027 \\
\hline Some college & -0.098 & 0.265 & -0.021 & -0.089 & 0.245 & -0.030 \\
\hline College graduation & -0.044 & 0.673 & -0.009 & 0.022 & 0.809 & 0.007 \\
\hline Employed & -0.142 & 0.086 & -0.029 & 0.099 & 0.145 & 0.033 \\
\hline Smoker & -0.093 & 0.136 & -0.019 & -0.137 & $0.012^{*}$ & -0.046 \\
\hline \multicolumn{7}{|l|}{ Region (Northeast) } \\
\hline Midwest & -0.113 & 0.261 & -0.025 & -0.053 & 0.520 & -0.018 \\
\hline South & -0.212 & $0.030 *$ & -0.046 & -0.140 & 0.101 & -0.046 \\
\hline West & -0.162 & 0.123 & -0.035 & 0.015 & 0.870 & 0.005 \\
\hline
\end{tabular}


Table 2 (Continued)

\begin{tabular}{|c|c|c|c|c|c|c|}
\hline \multirow{3}{*}{$\begin{array}{l}\text { Outcomes } \\
\text { Covariates (reference group) }\end{array}$} & \multicolumn{6}{|c|}{ Bivariate probit model (unweighted/weighted $N=9,332 / 2,091,774$ ) } \\
\hline & \multicolumn{3}{|c|}{ Hospital/ED visit } & \multicolumn{3}{|c|}{ PCP office visit } \\
\hline & Coefficient & P-value & Marginal effect & Coefficient & $P$-value & Marginal effect \\
\hline \multicolumn{7}{|l|}{ Comorbidities } \\
\hline Myocardial infarction & 0.302 & $0.00 I^{*}$ & 0.063 & 0.202 & $0.012^{*}$ & 0.067 \\
\hline Angina or coronary & 0.181 & $0.020 *$ & 0.038 & 0.208 & $0.003 *$ & 0.069 \\
\hline \multicolumn{7}{|l|}{ heart disease } \\
\hline Stroke & 0.032 & 0.704 & 0.007 & -0.092 & 0.233 & -0.030 \\
\hline Asthma & -0.022 & 0.718 & -0.005 & 0.048 & 0.364 & 0.016 \\
\hline Cancer & 0.033 & 0.617 & 0.007 & 0.073 & 0.184 & 0.024 \\
\hline Arthritis & -0.099 & 0.095 & -0.021 & 0.102 & 0.051 & 0.034 \\
\hline Depression & 0.035 & 0.567 & 0.007 & 0.069 & 0.229 & 0.023 \\
\hline Kidney disease & 0.168 & 0.078 & 0.035 & 0.087 & 0.313 & 0.029 \\
\hline Diabetes & 0.025 & 0.717 & 0.005 & 0.054 & 0.406 & 0.018 \\
\hline Year (20II) & 0.072 & 0.186 & 0.015 & 0.077 & 0.121 & 0.026 \\
\hline Constant & -0.850 & $<0.00 I^{*}$ & NA & -0.678 & $<0.00 I^{*}$ & NA \\
\hline
\end{tabular}

Note: $* P<0.05$. Rho $(\rho)=0.691 \quad(P<0.001)$.

Abbreviations: ED, emergency department; RHCs, rural health centers; FQHCs, federally qualified health centers; NA, not applicable; PCP, primary care physician.

respectively. An additional pulmonary care specialist per 100,000 persons reduced the likelihood of a hospital/ED visit by $0.4 \mathrm{pp}(P=0.028)$. An additional hospital per 100,000 persons increased the likelihood of hospital/ED visit by $0.8 \mathrm{pp}$ $(P=0.008)$. However, safety net facilities were not found to be related to hospital utilizations.

Health care utilizations were also associated with COPD medications, annual income, and comorbidities. Compared to those without any medications, additional usage of COPD medication increased the likelihood of hospital/ED visits and PCP office visits $(P<0.001)$. The low-income adults $(<\$ 15,000)$ demonstrated $6.0 \mathrm{pp}$ more likelihood of hospital/ ED visits than those with $\$ 35,000-\$ 49,999$ (reference group) income $(P=0.021)$. In contrast, the high-income adults $(\geq \$ 70,000)$ demonstrated $6.5 \mathrm{pp}$ less likelihood of hospital/ ED visits compared to the reference group $(P=0.008)$. Those with myocardial infarction and angina or coronary heart disease had a $6.3 \mathrm{pp}$ and $3.8 \mathrm{pp}$ more likelihood of hospital/ED visits and $6.7 \mathrm{pp}$ and $6.9 \mathrm{pp}$ more likelihood of PCP office visits, respectively (all $P<0.05$ ).

In addition, this study found that health care disparities appeared in race, sex, and age-groups. African-Americans had a $9.2 \mathrm{pp}$ and $8.5 \mathrm{pp}$ more likelihood of hospital/ED visits and PCP office visits compared to Caucasians, respectively (all $P<0.01$ ). Males showed a $3.6 \mathrm{pp}$ and $10.4 \mathrm{pp}$ less likelihood of hospital/ED visits and PCP office visits than that of females, respectively (all $P<0.01$ ). Older adults (aged 55+ years) demonstrated a 9.9-11.4 pp $(P<0.01)$ less likelihood of hospital/ED visits than younger adults (aged 18-34 years).
Sensitivity analyses in Table 3, based upon lagged values for county-level variables, showed consistent results with our findings in Table 2. Subpopulation analyses based on the presence of pulmonary care specialists within counties (Table 4) also supported our primary findings as mentioned earlier. In counties without any pulmonary care specialists, hospitals were positively associated with hospital/ED visits. In contrast, in counties with any pulmonary care specialists, only pulmonary care specialists were negatively associated with hospital/ED visits.

\section{Discussion}

Our study determined that the availability of pulmonary care specialists played a key role in reducing hospital utilizations among adults with COPD. An additional pulmonary care specialist per 100,000 persons may prevent hospital/ ED visit of 37 persons (weighted: 8,367 persons) among 9,332 samples (weighted: 2 million) by decreasing $0.4 \mathrm{pp}$ of likelihood of hospital/ED visit. On the contrary, availability of safety net facilities demonstrated no effect, and availability of hospitals actually served to increase hospital utilizations. An additional hospital per 100,000 persons may increase hospital/ED visit of 75 persons (weighted: 16,734 persons) among 9,332 samples (weighted: 2 million) by increasing $0.8 \mathrm{pp}$ of likelihood of hospital/ED visit. On the other hand, we did not find any association between access-to-care factors and PCP office visits. Our sensitivity and subpopulation analyses confirmed the findings, indicating that our results were robust over time and reliable in subpopulations. 
Table 3 Results of sensitivity analysis based on lagged values of county-level variables

\begin{tabular}{|c|c|c|c|c|c|c|}
\hline \multirow{2}{*}{$\begin{array}{l}\text { Outcomes } \\
\text { Covariates }\end{array}$} & \multicolumn{3}{|c|}{ Hospital/ED visit } & \multicolumn{3}{|c|}{ PCP office visit } \\
\hline & Coefficient & $P$-value & Marginal effect & Coefficient & $P$-value & Marginal effect \\
\hline \multicolumn{7}{|l|}{ All lagged variables } \\
\hline $\begin{array}{l}\text { Pulmonary care specialists } \\
\text { per } 100,000 \text { persons }\end{array}$ & -0.019 & $0.04 I^{*}$ & -0.004 & -0.005 & 0.589 & -0.002 \\
\hline RHCs per 100,000 persons & -0.003 & 0.642 & -0.001 & -0.003 & 0.529 & -0.001 \\
\hline FQHCs per 100,000 persons & 0.009 & 0.311 & 0.002 & 0.003 & 0.701 & 0.001 \\
\hline Hospitals per 100,000 persons & 0.036 & $0.037 *$ & 0.008 & NA & & \\
\hline PCPs per 10,000 persons & NA & & & -0.003 & 0.758 & -0.001 \\
\hline \multicolumn{7}{|c|}{ Lagged variables of pulmonary specialists and safety net facilities } \\
\hline Pulmonary care specialists & -0.019 & $0.042^{*}$ & -0.004 & -0.008 & 0.409 & -0.003 \\
\hline per 100,000 persons & & & & & & \\
\hline RHCs per 100,000 persons & -0.003 & 0.611 & -0.001 & -0.003 & 0.554 & -0.001 \\
\hline FQHCs per 100,000 persons & 0.008 & 0.314 & 0.002 & 0.003 & 0.705 & 0.001 \\
\hline Hospitals per 100,000 persons & 0.038 & $0.027 *$ & 0.008 & NA & & \\
\hline PCPs per 10,000 persons & NA & & & 0.002 & 0.822 & 0.001 \\
\hline \multicolumn{7}{|c|}{ Lagged variables of pulmonary specialists only } \\
\hline Pulmonary care specialists & -0.018 & $0.045^{*}$ & -0.004 & -0.008 & 0.419 & -0.003 \\
\hline per 100,000 persons & & & & & & \\
\hline RHCs per 100,000 persons & -0.002 & 0.740 & $-4.75 E-04$ & -0.002 & 0.642 & -0.001 \\
\hline FQHCs per 100,000 persons & 0.007 & 0.397 & 0.001 & 0.003 & 0.738 & 0.001 \\
\hline Hospitals per 100,000 persons & 0.038 & $0.031 *$ & 0.008 & NA & & \\
\hline PCPs per 10,000 persons & NA & & & 0.002 & 0.814 & 0.001 \\
\hline
\end{tabular}

Note: $* P<0.05$.

Abbreviations: ED, emergency department; RHCs, rural health centers; FQHCs, federally qualified health centers; NA, not applicable; PCP, primary care physician.

The inverse association of pulmonary care specialists with hospital utilization could be explained by inverse relationships between availability of pulmonary care specialists and ambulatory care sensitive condition. ${ }^{17,30}$ Pulmonary care specialists more stringently followed national guidelines for COPD care as compared to generalists in hospital, which served to improve health status and to reduce severe acute exacerbations, leading to a reduction of hospital utilization. ${ }^{31}$ In addition, primary care guided by respiratory care specialists also served to reduce hospital admissions. ${ }^{22}$
In contrast, the positive association of hospital availability with hospital utilization could be explained by an assumption that the number of hospitals is a proxy for distance to hospital, inferring that larger numbers of hospitals within a county results in the distance to a hospital being shorter ${ }^{26}$ The distance to a hospital is a barrier to access due to travel time, which is inversely associated with hospital utilization..$^{21,26}$ As such, increasing the number of hospitals will conceivably reduce the distance to hospital, leading to the increased utilization of hospitals. For example, adults

Table 4 Results of subpopulation analyses based on existence of pulmonary specialists

\begin{tabular}{|c|c|c|c|c|c|c|}
\hline \multirow{2}{*}{$\begin{array}{l}\text { Outcomes } \\
\text { Covariates }\end{array}$} & \multicolumn{3}{|c|}{ Hospital/ED visit } & \multicolumn{3}{|c|}{ PCP office visit } \\
\hline & Coefficient & $P$-value & Marginal effect & Coefficient & $P$-value & Marginal effect \\
\hline \multicolumn{7}{|c|}{ Number of pulmonary specialists $=0$ (unweighted/weighted $N=2,58 \mathrm{I} / 5 \mathrm{I}, 339$ ) } \\
\hline $\begin{array}{l}\text { Pulmonary care specialists } \\
\text { per } 100,000 \text { persons }\end{array}$ & NA & & & NA & & \\
\hline RHCs per 100,000 persons & -0.001 & 0.836 & $-3.06 \mathrm{E}-04$ & -0.005 & 0.409 & -0.002 \\
\hline FQHCs per 100,000 persons & 0.013 & 0.216 & 0.003 & 0.005 & 0.596 & 0.002 \\
\hline Hospitals per 100,000 persons & 0.056 & $0.005^{*}$ & 0.012 & NA & & \\
\hline PCPs per 10,000 persons & NA & & & 0.002 & 0.914 & 0.001 \\
\hline \multicolumn{7}{|c|}{ Number of pulmonary specialists $>0$, including pulmonary care specialist variable (unweighted/weighted $N=6,75 I / I, 579,435$ ) } \\
\hline Pulmonary care specialists & -0.028 & $0.016^{*}$ & -0.006 & -0.007 & 0.522 & -0.002 \\
\hline per 100,000 persons & & & & & & \\
\hline RHCs per 100,000 persons & -0.010 & 0.632 & -0.002 & -0.008 & 0.633 & -0.003 \\
\hline FQHCs per 100,000 persons & 0.001 & 0.888 & $2.76 \mathrm{E}-04$ & -0.005 & 0.563 & -0.002 \\
\hline Hospitals per 100,000 persons & 0.026 & 0.369 & 0.005 & NA & & \\
\hline PCPs per 10,000 persons & NA & & & 0.003 & 0.805 & 0.001 \\
\hline
\end{tabular}

Note: $* p<0.05$.

Abbreviations: ED, emergency department; RHCs, rural health centers; FQHCs, federally qualified health centers; NA, not applicable; PCP, primary care physician. 
with hospital/ED visits had more available hospitals (2.27) as compared to those (2.03) without hospital/ED visits $(P=0.037)$, possibly leading to reduced distance to hospital. Therefore, an additional unit of hospital may make distance to hospital (or travel time) shorter, leading to an increase in the likelihood of hospitalization.

As an external validity, our results were partially consistent with the previous study. ${ }^{17}$ Jackson et $\mathrm{al}^{17}$ found that counties with pulmonary care specialists were $26 \%-37 \%$ less likely to receive hospitalizations $(P<0.05)$, which is qualitatively consistent with our results. They also found that safety net facilities demonstrated ambiguous associations with hospitalizations. ${ }^{17}$ Hospitalization rates were lowest among counties with only FQHCs and highest among counties with only RHCs, which were not consistent with our results. ${ }^{17}$ In a supplementary analysis (Table S2) with categorized variable of safety net facilities (1: no safety net facility, 2: RHC only, 3: FQHC only, and 4: both), no association was found between safety net facilities and hospital/ED visits.

Our results on demographic and socioeconomic factors were consistent with previous studies. Adults with low income $(<\$ 15,000)$ or some form of comorbidities were vulnerable to hospitalization. ${ }^{8-12}$ Older age-groups were less likely to have hospital/ED visits, while the previous study demonstrated that older age-groups had more hospitalizations. ${ }^{17}$ Our result on the age-group is not contradictory to the previous study because our study population is different from that of the previous study. That is, our study included hospitalized and nonhospitalized adults with COPD, while the previous study counted only the number of hospitalized patients with COPD. When we counted adults with hospital/ED visits, the number of adults (339-407) with hospital/ED visits among older age-groups (aged 55+ years) were higher than that of adults (169) with hospital/ED visits among younger age-group (aged 18-34 years), consistent with the previous study.

In terms of the degree of influences on hospital/ED visits, several covariates were more influential on the reduction of likelihood of the hospital/ED visits than the pulmonary care specialist. For instance, compared to the pulmonary care specialist ( $0.4 \mathrm{pp}$ reduction), old agegroups (55-64 years and 65+ years), high-income group ( $\geq \$ 70,000$ ), and widows showed 9.9-11.4 pp, 6.5 pp, and $3.5 \mathrm{pp}$ less likelihood of hospital/ED visits than the reference groups (18-34 age-group, \$35,000-\$49,999 income level, and married group), respectively. However, the pulmonary care specialist is a more appropriate policy tool to have an influence on hospital/ED visits than those covariates since it is hard to change those demographic and socioeconomic variables.

\section{Limitations}

This study has a number of limitations. First, large missing values in covariates may affect our results. But, no major selection bias was found since missing in covariates seems to be random when we compared demographics between adults with outcomes and those without outcomes. Second, the availability of access-to-care factors in nearby counties may weaken our results because patients may travel to see pulmonary care specialists. The increased travel time still may be a barrier to access resulting in making patients less likely to see specialists in nearby counties. Third, the high proportions of subjects with COPD aged $<45$ years $(\sim 22 \%)$ and of female patients (45\%) might be little different from other hospital dataset.

\section{Conclusion and policy implications}

In conclusion, the availability of pulmonary care specialists was a key access-to-care factor to reduce hospital utilizations among adults with COPD, yet accessibility of hospital increases hospital utilizations. These findings could provide the following implications: the first implication is that the increase in the availability of pulmonary care specialists to counties with no or low access to pulmonary specialists may lessen hospitalizations, leading to reduced costs. However, since there is limited numbers of pulmonary care specialists, usage of telehealth services might be an alternative to increase availability. ${ }^{32}$ The second implication is that since the availability of hospitals actually increases hospital utilizations, directing patients with COPD to pulmonary care specialists, and alternative COPD treatment centers may serve to reduce hospital utilizations. ${ }^{17}$

\section{Acknowledgment}

We acknowledge the Research Open Access Publishing (ROAAP) Fund of the University of Illinois at Chicago for financial support towards the open access publishing fee for this article.

\section{Author contributions}

All authors contributed toward data analysis, drafting and critically revising the paper and agree to be accountable for all aspects of the work.

\section{Disclosure}

The authors report no conflicts of interest in this work. 


\section{References}

1. Murphy SL, Xu J, Kochanek KD. Deaths: final data for 2010. Natl Vital Stat Rep. 2013;61(4):1-117.

2. Sullivan SD, Ramsey SD, Lee TA. The economic burden of COPD. Chest. 2000;117(2 suppl):5S-9S.

3. Halpern MT, Stanford RH, Borker R. The burden of COPD in the U.S.A.: results from the Confronting COPD survey. Respir Med. 2003; 97(suppl C):S81-S89.

4. Blanchette CM, Gross NJ, Altman P. Rising costs of COPD and the potential for maintenance therapy to slow the trend. Am Health Drug Benefits. 2014;7(2):98-106.

5. U.S. Department of Health and Human Services, Office of Disease Prevention and Health Promotion [webpage on the Internet]. Healthy People 2020. Available from: http://www.healthypeople.gov/2020/ topics-objectives/topic/respiratory-diseases/objectives. Accessed August 25, 2015.

6. Miravitlles M. Health economic consequences of COPD exacerbations. In: Wedzicha JA, Martinez FJ, editors. Chronic Obstructive Pulmonary Disease Exacerbations. New York, NY: Informa Healthcare; 2009: 225-232.

7. Bourbeau J, Julien M, Maltais F, et al; Chronic Obstructive Pulmonary Disease axis of the Respiratory Network Fonds de la Recherche en Santé du Québec. Reduction of hospital utilization in patients with chronic obstructive pulmonary disease: a disease-specific self-management intervention. Arch Intern Med. 2003;163(5):585-591.

8. Bahadori K, FitzGerald JM. Risk factors of hospitalization and readmission of patients with COPD exacerbation - systematic review. Int J Chron Obstruct Pulmon Dis. 2007;2(3):241-251.

9. Mullerova H, Maselli DJ, Locantore N, et al; ECLIPSE Investigators. Hospitalized exacerbations of COPD: risk factors and outcomes in the ECLIPSE cohort. Chest. 2015;147(4):999-1007.

10. Niewoehner DE, Lokhnygina Y, Rice K, et al. Risk indexes for exacerbations and hospitalizations due to COPD. Chest. 2007;131(1):20-28.

11. Garcia-Aymerich J, Monso E, Marrades RM, et al; EFRAM Investigators. Risk factors for hospitalization for a chronic obstructive pulmonary disease exacerbation. EFRAM study. Am J Respir Crit Care Med. 2001;164(6):1002-1007.

12. Xu W, Collet JP, Shapiro S, et al. Independent effect of depression and anxiety on chronic obstructive pulmonary disease exacerbations and hospitalizations. Am J Respir Crit Care Med. 2008;178(9):913-920.

13. Mapel DW, Hurley JS, Frost FJ, Petersen HV, Picchi MA, Coultas DB. Health care utilization in chronic obstructive pulmonary disease. A case-control study in a health maintenance organization. Arch Intern Med. 2000;160(17):2653-2658.

14. Moolgavkar SH. Air pollution and hospital admissions for chronic obstructive pulmonary disease in three metropolitan areas in the United States. Inhal Toxicol. 2000;12(suppl 4):75-90.

15. Medina-Ramon M, Zanobetti A, Schwartz J. The effect of ozone and PM10 on hospital admissions for pneumonia and chronic obstructive pulmonary disease: a national multicity study. Am J Epidemiol. 2006; 163(6):579-588.

16. Hahn EJ, Rayens MK, Adkins S, Simpson N, Frazier S, Mannino DM. Fewer hospitalizations for chronic obstructive pulmonary disease in communities with smoke-free public policies. Am J Public Health. 2014;104(6):1059-1065.

17. Jackson BE, Suzuki S, Coultas D, et al. Safety-net facilities and hospitalization rates of chronic obstructive pulmonary disease: a cross-sectional analysis of the 2007 Texas Health Care Information Council inpatient data. Int J Chron Obstruct Pulmon Dis. 2011;6:563-571.
18. Fan VS, Bridevaux PO, McDonell MB, Fihn SD, Besser LM, Au DH. Regional variation in health status among chronic obstructive pulmonary disease patients. Respiration. 2011;81(1):9-17.

19. Goins RT, Williams KA, Carter MW, Spencer SM, Solovieva T. Perceived barriers to health care access among rural older adults: a qualitative study. J Rural Health. 2005;21(3):206-213.

20. Parchman ML, Culler SD. Preventable hospitalizations in primary care shortage areas - an analysis of vulnerable medicare beneficiaries. Arch Fam Med. 1999;8(6):487-491.

21. Goodman DC, Fisher E, Stukel TA, Chang CH. The distance to community medical care and the likelihood of hospitalization: is close always better? Am J Public Health. 1997;87(7):1144-1150.

22. Hull S, Mathur R, Lloyd-Owen S, Round T, Robson J. Improving outcomes for people with COPD by developing networks of general practices: evaluation of a quality improvement project in east London. NPJ Prim Care Respir Med. 2014;24:14082.

23. Fromer L. Implementing chronic care for COPD: planned visits, care coordination, and patient empowerment for improved outcomes. Int $J$ Chron Obstruct Pulmon Dis. 2011;6:605-614.

24. Zhang W, Mueller KJ, Chen LW, Conway K. The role of rural health clinics in hospitalization due to ambulatory care sensitive conditions: a study in Nebraska. J Rural Health. 2006;22(3):220-223.

25. Probst JC, Laditka JN, Laditka SB. Association between community health center and rural health clinic presence and county-level hospitalization rates for ambulatory care sensitive conditions: an analysis across eight US states. BMC Health Serv Res. 2009;9:134.

26. Sanz-Barbero B, Garcia LO, Hernandez TB. The effect of distance on the use of emergency hospital services in a Spanish region with high population dispersion: a multilevel analysis. Med Care. 2012;50(1):27-34.

27. U.S. Department of Health and Human Services, Centers for Disease Control and Prevention, editor. Behavioral Risk Factor Surveillance System Survey (BRFSS) Data. Atlanta, GA: U.S. Department of Health and Human Services, Centers for Disease Control and Prevention; 2011-2012.

28. U.S. Department of Health and Human Services, Health Resources and Services Administration, Bureau of Health Workforce, editor. Area Health Resources File (AHRF). Rockville, MD: U.S. Department of Health and Human Services, Health Resources and Services Administration, Bureau of Health Workforce; 2013-2014.

29. Greene WH. Econometric Analysis. 7th ed. Boston, MA: Prentice Hall; 2012.

30. Laditka JN, Laditka SB, Probst JC. More may be better: evidence of a negative relationship between physician supply and hospitalization for ambulatory care sensitive conditions. Health Serv Res. 2005;40(4): 1148-1166.

31. Pothirat C, Liwsrisakun C, Bumroongkit C, Deesomchok A, Theerakittikul T, Limsukon A. Comparative study on health care utilization and hospital outcomes of severe acute exacerbation of chronic obstructive pulmonary disease managed by pulmonologists vs internists. Int J Chron Obstruct Pulmon Dis. 2015;10:759-766.

32. Raza T, Joshi M, Schapira RM, Agha Z. Pulmonary telemedicine - a model to access the subspecialist services in underserved rural areas. Int J Med Inform. 2009;78(1):53-59. 


\section{Supplementary materials}

Table SI Correlation between outcome variables

\begin{tabular}{lll}
\hline & Hospital/ED visit & PCP office visit \\
\hline Hospital/ED visit & 1.00 & 1.00 \\
PCP office visit & $0.40(P<0.001)$ & \\
\hline
\end{tabular}

Abbreviations: ED, emergency department; PCP, primary care physician.

Table S2 Results of supplementary analysis based on safety net facilities categorical variable

\begin{tabular}{|c|c|c|c|c|c|c|}
\hline \multirow{3}{*}{$\begin{array}{l}\text { Outcomes } \\
\text { Covariates (reference group) }\end{array}$} & \multicolumn{6}{|c|}{ All samples (unweighted/weighted $N=9,332 / 2,091,774$ ) } \\
\hline & \multicolumn{3}{|c|}{ Hospital/ED visit } & \multicolumn{3}{|c|}{ PCP office visit } \\
\hline & Coefficient & $P$-value & Marginal effect & Coefficient & $P$-value & Marginal effect \\
\hline $\begin{array}{l}\text { Pulmonary care specialists } \\
\text { per } 100,000 \text { persons }\end{array}$ & -0.020 & $0.029 *$ & -0.004 & -0.006 & 0.482 & -0.002 \\
\hline \multicolumn{7}{|l|}{ Safety net facilities (no facility) } \\
\hline $\mathrm{RHCs}$ and $\mathrm{FQHCs}$ & 0.066 & 0.489 & 0.014 & 0.097 & 0.315 & 0.032 \\
\hline RHCs only & -0.042 & 0.726 & -0.008 & -0.014 & 0.909 & -0.004 \\
\hline FQHCs only & 0.030 & 0.758 & 0.006 & 0.031 & 0.755 & 0.010 \\
\hline Hospitals per 100,000 persons & 0.040 & $0.016 *$ & 0.008 & NA & & \\
\hline PCPs per 10,000 persons & NA & & & 0.002 & 0.826 & 0.001 \\
\hline
\end{tabular}

Note: $* P<0.05$.

Abbreviations: ED, emergency department; RHCs, rural health centers; FQHCs, federally qualified health centers; NA, not applicable; PCP, primary care physician.

\section{Publish your work in this journal}

The International Journal of COPD is an international, peer-reviewed journal of therapeutics and pharmacology focusing on concise rapid reporting of clinical studies and reviews in COPD. Special focus is given to the pathophysiological processes underlying the disease, intervention programs, patient focused education, and self management protocols

\section{Dovepress}

This journal is indexed on PubMed Central, MedLine and CAS. The manuscript management system is completely online and includes a very quick and fair peer-review system, which is all easy to use. Visit http://www.dovepress.com/testimonials.php to read real quotes from published authors. 